ASC Report No. $47 / 2009$

\title{
Neumann Problems with Time Singularities
}

Irena Rachůnková, Svatoslav Staněk, Ewa Weinmüller, Michael Zenz 


\section{Most recent ASC Reports}

46/2009 Kazuo Aoki, Ansgar Jüngel, Peter A. Markovich

Small Velocity and finite Temperature Variations in Kinetic Relaxation Models

45/2009 Ansgar Jüngel, Jan-Frederik Mennemann

Time-dependent Simulations of Multidimensional Quantum Waveguides Using a Time-Splitting Spectral method

44/2009 Markus Aurada, Michael Ebner, Samuel Ferraz-Leite, Petra Goldonits, Michael Karkulik, Markus Mayr, Dirk Praetorius

HILBERT - A MATLAB Implementation of Adaptive BEM

43/2009 Matthias Langer, Harald Woracek

A Local Inverse Spectral Theorem for Hamilton Systems

42/2009 Ansgar Jüngel

Energy Transport in Semiconductor Devices

41/2009 Ansgar Jüngel

Global Weak Solutions to Compressible Navier-Stokes Equations for Quantum Fluid

40/2009 Markus Aurada, Petra Goldenits, Dirk Praetorius

Convergence of Data Perturbed Adaptive Boundary Element Methods

39/2009 Ilona Gucwa, Peter Szmolyan

Scaling in Singular Perturbation Problems: Blowing-up a Relaxation Oscillator

38/2009 Anton Baranov, Harald Woracek

Majorization in de Branges Spaces III. Division of Blaschke Products

37/2009 Philipp Dörsek, Jens Markus Melenk

Adaptive $h p$-FEM for the Contact Problem with Tresca Friction in Linear Elasticity: The Primal-dual Formulation and a Posteriori Error Estimation

Institute for Analysis and Scientific Computing

Vienna University of Technology

Wiedner Hauptstraße 8-10

1040 Wien, Austria

E-Mail: admin@asc.tuwien.ac.at

WWW: http://www.asc.tuwien.ac.at

FAX: $\quad+43-1-58801-10196$

ISBN 978-3-902627-02-5

(C) Alle Rechte vorbehalten. Nachdruck nur mit Genehmigung des Autors.

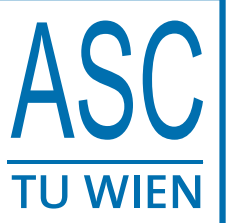




\title{
Neumann problems with time singularities
}

\author{
Irena Rachůnková, Svatoslav Staněk, Ewa Weinmüller, Michael Zenz \\ Department of Mathematical Analysis, Faculty of Science, \\ Palacký University, tř. 17. listopadu 12, 77146 Olomouc, Czech Republic \\ e-mail: rachunko@inf.upol.cz stanek@inf.upol.cz \\ Institute for Analysis and Scientific Computing, \\ Vienna University of Technology, \\ Wiedner Hauptstrasse 8-10, A-1040 Wien, Austria \\ e-mail: e.weinmueller@tuwien.ac.at m.zenz@gmx.com
}

Abstract: In this paper we study the existence and uniqueness of solutions to a nonlinear Neumann problem for a scalar second order ordinary differential equation

$$
u^{\prime \prime}=\frac{a}{t} u^{\prime}+f\left(t, u, u^{\prime}\right)
$$

where $a \in \mathbb{R} \backslash\{0\}$, and $f(t, x, y)$ satisfies the $L_{p}$-Carathéodory conditions on $[0, T] \times \mathbb{R}^{2}$ for some $p>1$.

Key words: Singular boundary value problem, Neumann problem, time singularity of the first kind, existence and uniqueness of solution, collocation methods, lower and upper functions

2000 Mathematics Subject Classification: 34B16, 34B18

\section{Motivation}

The aim of this work is to apply the theory of limit properties of functions satisfying singular differential equations developed in [28] to show the existence and uniqueness of solutions to a nonlinear Neumann problem exhibiting a singularity of the first kind in time. In many applications, second order singular models, cf. [4], [5], [10], [19], and [30], assume the forms

$$
u^{\prime \prime}=\frac{a_{1}}{t^{\alpha}} u^{\prime}+\frac{a_{0}}{t^{\alpha+1}} u+f\left(t, u, u^{\prime}\right), \quad u^{\prime \prime}=\frac{a}{t^{\alpha}} u^{\prime}+f\left(t, u, u^{\prime}\right), \quad t>0,
$$

where $a_{1}, a_{0}, a$ and $f$ are given. We say that for $\alpha=1$ the problem exhibits a singularity of the first kind at $t=0$, while for $\alpha>1$, the singularity is essential 
or of the second kind. In [30], the existence and uniqueness results in case of smooth data function $f$ has been developed. This analysis is based on techniques proposed in [12]. However, in applications mentioned below this smoothness assumption does not hold and therefore, there is a need for covering the case of unsmooth inhomogeneities $f$.

Here, we consider differential equations with a singularity of the first kind, $\alpha=1$, of the form

$$
u^{\prime \prime}=\frac{a}{t} u^{\prime}+f\left(t, u, u^{\prime}\right)
$$

where $a \in \mathbb{R} \backslash\{0\}$, and the function $f(t, x, y)$ is defined for a.e. $t \in[0, T]$ and for all $(x, y) \in \mathcal{D} \subset \mathbb{R} \times \mathbb{R}$. Clearly, the above equation is singular at $t=0$ because of the first term in the right-hand side, which is in general unbounded for $t \rightarrow 0$. Moreover, we also alow the function $f$ to be unbounded or bounded but discontinuous for certain values of the time variable $t \in[0, T]$. This form of $f$ is motivated by a variety of initial and boundary value problems known from applications and having nonlinear, discontinuous forcing terms, such as electronic devices which are often driven by square waves or more complicated discontinuous inputs. Typically, such problems are modelled by differential equations where $f$ has jump discontinuities at a discrete set of points in $(0, T)$, cf. [23]. Many other applications, cf. [1]-[11], [16], [19], [24]-[29] also show these structural difficulties.

New technique developed in [28] which we will apply in this paper, enables to extend results from [21] and [30] based on ideas presented in [12], where, as already mentioned, problems of the above form but with appropriately smooth data function $f$ have been discussed.

\section{Introduction}

The following notation will be used throughout the paper. Let $J \subset \mathbb{R}$ be an interval. Then, we denote by $L_{1}(J)$ the set of functions which are (Lebesgue) integrable on $J$. The corresponding norm is $\|u\|_{1}:=\int_{J}|u(t)| \mathrm{d} t$. Let $p>1$. By $L_{p}(J)$, we denote the set of functions whose $p$-th powers of modulus are integrable on $J$ with the corresponding norm given by $\|u\|_{p}:=\left(\int_{J}|u(t)|^{p} \mathrm{~d} t\right)^{1 / p}$.

Moreover, let us by $C(J)$ and $C^{1}(J)$ denote the sets of functions being continuous on $J$, and having continuous first derivatives on $J$, respectively. The norm on $C[0, T]$ is defined as $\|u\|_{\infty}:=\max _{t \in[0, T]}\{|u(t)|\}$.

Finally, we denote by $A C(J)$ and $A C^{1}(J)$ the sets of functions which are absolutely continuous on $J$, and which have absolutely continuous first derivatives on $J$, respectively. Analogously, $A C_{l o c}(J)$ and $A C_{l o c}^{1}(J)$ are the sets of functions being absolutely continuous on each compact subinterval $I \subset J$, and having 
absolutely continuous first derivatives on each compact subinterval $I \subset J$, respectively.

As already said, we investigate differential equations of the form

$$
u^{\prime \prime}(t)=\frac{a}{t} u^{\prime}(t)+f\left(t, u(t), u^{\prime}(t)\right) \text { a.e. on }[0, T],
$$

where $a \in \mathbb{R} \backslash\{0\}$. For the subsequent analysis we assume that there exists $p>1$ such that

$$
f \text { satisfies the } L_{p} \text { - Carathéodory conditions on }[0, T] \times \mathbb{R} \times \mathbb{R} \text {, }
$$

specified in the following definition.

Definition 2.1. Let $p>1$. A function $f$ satisfies the $L_{p}$-Carathéodory conditions on the set $[0, T] \times \mathbb{R} \times \mathbb{R}$ if

(i) $f(\cdot, x, y):[0, T] \rightarrow \mathbb{R}$ is measurable for all $(x, y) \in \mathbb{R} \times \mathbb{R}$,

(ii) $f(t, \cdot, \cdot): \mathbb{R} \times \mathbb{R} \rightarrow \mathbb{R}$ is continuous for a.e. $t \in[0, T]$,

(iii) for each compact set $\mathcal{K} \subset \mathbb{R} \times \mathbb{R}$ there exists a function $m_{\mathcal{K}}(t) \in L_{p}[0, T]$ such that $|f(t, x, y)| \leq m_{\mathcal{K}}(t)$ for a.e. $t \in[0, T]$ and all $(x, y) \in \mathcal{K}$.

In [28], a description of an asymptotical behavior for $t \rightarrow 0+$ of functions $u$ satisfying (2.1) a.e. on $[0, T]$ is given. Such functions $u$ will be called solutions of (2.1) if they additionally satisfy the smoothness requirement $u \in A C^{1}[0, T]$, see next definition.

Definition 2.2. A function $u:[0, T] \rightarrow \mathbb{R}$ is called a solution of equation (2.1) if $u \in A C^{1}[0, T]$ and

$$
u^{\prime \prime}(t)=\frac{a}{t} u^{\prime}(t)+f\left(t, u(t), u^{\prime}(t)\right)
$$

holds a.e. on $[0, T]$.

By applying the theory from [28], we will provide existence and/or uniqueness results for solutions of equation (2.1) subject to the Neumann boundary conditions $u^{\prime}(0)=u^{\prime}(T)=0$. The paper is organized as follows. In Section 3 we recapitulate main analytical results from [28] and give their generalizations necessary for further investigations. The Neumann problem is then analyzed in Section 4. Finally, in Section 5, we illustrate the theoretical findings by means of numerical experiments. 


\section{Limit properties of functions satisfying singu- lar equations}

First, we consider the linear equation, $a \in \mathbb{R} \backslash\{0\}$,

$$
u^{\prime \prime}(t)=\frac{a}{t} u^{\prime}(t)+h(t) \text { a.e. on }(0, T],
$$

where $h \in L_{p}[0, T]$ and $p>1$.

Main results dealing with limit properties in context of the linear equation (3.1) are now formulated in the following two lemmas, cf. [28].

Lemma 3.1. Let $a>0$ and let $u \in A C_{l o c}^{1}(0, T]$ satisfy equation (3.1) a.e. on $[0, T]$. Then

$$
\lim _{t \rightarrow 0+} u(t) \in \mathbb{R}, \quad \lim _{t \rightarrow 0+} u^{\prime}(t)=0 .
$$

Moreover, $u$ can be extended to the whole interval $[0, T]$ in such a way that $u \in$ $A C^{1}[0, T]$.

Lemma 3.2. Let $a<0$ and let a function $u \in A C_{l o c}^{1}(0, T]$ satisfy equation (3.1) a.e. on $[0, T]$. For $a \in(-1,0)$, only one of the following properties holds,

(i) $\lim _{t \rightarrow 0+} u(t) \in \mathbb{R}, \quad \lim _{t \rightarrow 0+} u^{\prime}(t)=0$,

(ii) $\lim _{t \rightarrow 0+} u(t) \in \mathbb{R}, \quad \lim _{t \rightarrow 0+} u^{\prime}(t)= \pm \infty$.

For $a \in(-\infty,-1]$, u satisfies only one of the following properties:

(i) $\lim _{t \rightarrow 0+} u(t) \in \mathbb{R}, \quad \lim _{t \rightarrow 0+} u^{\prime}(t)=0$,

(ii) $\lim _{t \rightarrow 0+} u(t)=\mp \infty, \lim _{t \rightarrow 0+} u^{\prime}(t)= \pm \infty$.

In particular, $u$ can be extended to the whole interval $[0, T]$ with $u \in A C^{1}[0, T]$ if and only if $\lim _{t \rightarrow 0+} u^{\prime}(t)=0$.

Now, we consider the nonlinear equation (2.1), where $f$ has a global majorant in $L_{p}[0, T]$. This means that there exists a function $g \in L_{p}[0, T]$ such that

$$
|f(t, x, y)| \leq g(t) \text { for a.e. } t \in[0, T] \text { and all }(x, y) \in \mathbb{R} \times \mathbb{R} \text {. }
$$

Example 3.3. Let $v \in C\left(\mathbb{R}^{2}\right)$ be bounded and let $r \in L_{p}[0, T], p>1$. Then the functions

$$
f_{1}(t, x, y)=r(t) v(x, y), \quad f_{2}(t, x, y)=r(t)+v(x, y)
$$

satisfy (3.2) with $f=f_{j}, j=1,2$. 
As a consequence of Lemmas 3.1 and 3.2, we can now formulate main results for the nonlinear singular equation (2.1).

Theorem 3.4. Let us assume that (2.2) and (3.2) hold. Let $a>0$ and let $u \in A C_{l o c}^{1}(0, T]$ satisfy equation (2.1) a.e. on $[0, T]$. Then

$$
\lim _{t \rightarrow 0+} u(t) \in \mathbb{R}, \quad \lim _{t \rightarrow 0+} u^{\prime}(t)=0,
$$

and $u$ can be extended on $[0, T]$ in such a way that $u \in A C^{1}[0, T]$.

Proof. Assume that $u \in A C_{l o c}^{1}(0, T]$ satisfies equation (2.1) a.e. on $[0, T]$ and set $h(t):=f\left(t, u(t), u^{\prime}(t)\right)$ for a.e. $t \in[0, T]$. Then, by $(2.2), h \in L_{p}[\rho, T]$ for each $\rho \in(0, T]$ and, by $(3.2),|h(t)| \leq g(t)$ for a.e. $t \in[0, T]$. Hence $h \in L_{p}[0, T]$. Since the equality $u^{\prime \prime}(t)=a u^{\prime}(t) / t+h(t)$ holds a.e. on $[0, T]$, the result follows immediately due to Lemma 3.1.

Theorem 3.5. Let us assume (2.2) and (3.2) to hold. Let $a<0$ and let $u \in$ $A C_{l o c}^{1}(0, T]$ satisfy equation $(2.1)$ a.e. on $(0, T]$. If $a \in(-1,0)$, then $u$ satisfies exactly one of the following properties:

(i) $\lim _{t \rightarrow 0+} u(t) \in \mathbb{R}, \quad \lim _{t \rightarrow 0+} u^{\prime}(t)=0$,

(ii) $\lim _{t \rightarrow 0+} u(t) \in \mathbb{R}, \quad \lim _{t \rightarrow 0+} u^{\prime}(t)= \pm \infty$.

If $a \in(-\infty,-1]$ then $u$ satisfies exactly one of the following properties:

(i) $\lim _{t \rightarrow 0+} u(t) \in \mathbb{R}, \lim _{t \rightarrow 0+} u^{\prime}(t)=0$,

(ii) $\lim _{t \rightarrow 0+} u(t)=\mp \infty, \lim _{t \rightarrow 0+} u^{\prime}(t)= \pm \infty$.

In particular, $u$ can be extended on $[0, T]$ with $u \in A C^{1}[0, T]$ if and only if $\lim _{t \rightarrow 0+} u^{\prime}(t)=0$.

Proof. Let $h \in L_{p}[0, T]$ be as in the proof of Theorem 3.4. Then the result follows by Lemma 3.2.

The following corollary will be used in the next section.

Corollary 3.6. Let us assume that condition (2.2) holds. Let $a \neq 0$ and let $u \in A C_{l o c}^{1}(0, T]$ satisfy equation $(2.1)$ a.e. on $[0, T]$. Let us also assume that

$$
\sup \left\{|u(t)|+\left|u^{\prime}(t)\right|: t \in(0, T]\right\}<\infty .
$$

holds. Then

$$
\lim _{t \rightarrow 0+} u(t) \in \mathbb{R}, \quad \lim _{t \rightarrow 0+} u^{\prime}(t)=0,
$$

and $u$ can be extended on $[0, T]$ in such a way that $u \in A C^{1}[0, T]$. 
Proof. Let $\tilde{f}(t, x, y):=f\left(t, u(t), u^{\prime}(t)\right)$ for a.e. $t \in[0, T]$ and all $x, y \in \mathbb{R}$. Clearly

$$
u^{\prime \prime}(t)=\frac{a}{t} u^{\prime}(t)+\tilde{f}\left(t, u(t), u^{\prime}(t)\right)
$$

holds a.e. on $[0, T]$. By (2.2)and (3.3), there exists a function $g \in L_{p}[0, T]$ such that $|\tilde{f}(t, x, y)| \leq g(t)$ for a.e. $t \in[0, T]$ and all $(x, y) \in \mathbb{R} \times \mathbb{R}$. The results now follow from Theorems 3.4 and 3.5, where in equation (2.1) $f$ is replaced by $\tilde{f}$.

\section{Neumann Problem}

Using results formulated in Corollary 3.6 and the Fredholm-type existence theorem (see e.g. [22], [26], [27]), we are now in the position to show the existence and/or uniqueness of solutions of the nonlinear singular Neumann boundary value problem,

$$
\begin{aligned}
& u^{\prime \prime}(t)=\frac{a}{t} u^{\prime}(t)+f\left(t, u(t), u^{\prime}(t)\right) \text { a.e. on }[0, T] \\
& u^{\prime}(0)=0, \quad u^{\prime}(T)=0 .
\end{aligned}
$$

Definition 4.1. A function $u \in A C^{1}[0, T]$ is called a solution of the boundary value problem (4.1), if $u$ satisfies equation (4.1a) for a.e. $t \in[0, T]$ and the Neumann conditions (4.1b).

First, we consider the uniqueness.

Theorem 4.2. (Uniqueness) Let $a<0$ and let us assume that condition (2.2) holds. Moreover, let us assume that for each compact set $\mathcal{K} \subset \mathbb{R} \times \mathbb{R}$ there exists a nonnegative function $h_{\mathcal{K}} \in L_{1}[0, T]$ such that

$$
x_{1}>x_{2} \Rightarrow f\left(t, x_{1}, y_{1}\right)-f\left(t, x_{2}, y_{2}\right)>-h_{\mathcal{K}}(t)\left|y_{1}-y_{2}\right|
$$

for a.e. $t \in[0, T]$ and all $\left(x_{1}, y_{1}\right),\left(x_{2}, y_{2}\right) \in \mathcal{K}$. Then problem (4.1) has at most one solution.

Proof. Let $u_{1}$ and $u_{2}$ be different solutions of problem (4.1). Since $u_{1}, u_{2} \in$ $A C^{1}[0, T]$, there exists a compact set $\mathcal{K} \subset \mathbb{R} \times \mathbb{R}$ such that $\left(u_{i}(t), u_{i}^{\prime}(t)\right) \in \mathcal{K}$ for $t \in[0, T]$. Again, $v(t):=u_{1}(t)-u_{2}(t)$ for $t \in[0, T]$. Then

$$
v^{\prime}(0)=0, \quad v^{\prime}(T)=0 .
$$

We consider two cases.

Case 1. Assume that $u_{1}$ and $u_{2}$ have an intersection point. This means that there exists $t_{0} \in[0, T]$ such that $v\left(t_{0}\right)=0$. Since $u_{1}$ and $u_{2}$ are different, there exists $t_{1} \in[0, T], t_{1} \neq t_{0}$, such that $v\left(t_{1}\right) \neq 0$. 
(i) Let $t_{1}>t_{0}$. We can assume that $v\left(t_{1}\right)>0$ and define $v:=u_{2}-u_{1}$ otherwise. Then we can find $a_{0} \in\left(t_{0}, t_{1}\right)$ satisfying $v(t)>0$ for $t \in\left[a_{0}, t_{1}\right]$ and $v^{\prime}\left(a_{0}\right)>0$. Let $b_{0} \in\left(a_{0}, T\right]$ be the first zero of $v^{\prime}$. Then, if we set $[\alpha, \beta]:=\left[a_{0}, b_{0}\right]$, we see that

$$
v(t)>0 \text { for } t \in[\alpha, \beta], v^{\prime}(t)>0 \text { for } t \in[\alpha, \beta), v^{\prime}(\beta)=0 .
$$

Now, by (4.1a), (4.2) and (4.4), we obtain

$$
v^{\prime \prime}(t)>\left(\frac{a}{t}-h_{\mathcal{K}}(t)\right) v^{\prime}(t) \text { for a.e. } t \in[\alpha, \beta]
$$

Denote by $h^{*}(t):=\frac{a}{t}-h_{\mathcal{K}}(t)$. Then $h^{*} \in L_{1}[\alpha, \beta]$ and $v^{\prime \prime}(t)-h^{*}(t) v^{\prime}(t)>0$ for a.e. $t \in[\alpha, \beta]$. Consequently,

$$
\left(v^{\prime}(t) \exp \left(-\int_{\alpha}^{t} h^{*}(s) d s\right)\right)^{\prime}>0 \text { for a.e. } t \in[\alpha, \beta] .
$$

Integrating the last inequality in $[\alpha, \beta]$, we obtain

$$
v^{\prime}(\beta) \exp \left(-\int_{\alpha}^{\beta} h^{*}(s) d s\right)>v^{\prime}(\alpha)>0
$$

which contradicts $v^{\prime}(\beta)=0$.

(ii) Let $v=0$ on $\left[t_{0}, T\right]$. Since $u_{1}$ and $u_{2}$ are different, we can find $\beta \in\left(0, t_{0}\right)$ such that (without loss of generality) $v(\beta)>0$, and $v^{\prime}(\beta)<0$. Due to (4.3) it is possible to find $\alpha \in[0, \beta)$ such that

$$
v(t)>0 \text { for } t \in[\alpha, \beta], v^{\prime}(t)<0 \text { for } t \in(\alpha, \beta], v^{\prime}(\alpha)=0 \text {. }
$$

Now, we conclude from (4.1a), (4.2) and $a<0$,

$$
v^{\prime \prime}(t)>\frac{a}{t} v^{\prime}(t)-h_{\mathcal{K}}(t)\left|v^{\prime}(t)\right| \geq h_{\mathcal{K}}(t) v^{\prime}(t) \text { for a.e. } t \in[\alpha, \beta]
$$

As above, we modify the last inequality, integrate it and obtain

$$
v^{\prime}(\beta) \exp \left(-\int_{\alpha}^{\beta} h_{\mathcal{K}}(s) d s\right)>v^{\prime}(\alpha)=0,
$$

which contradicts $v^{\prime}(\beta)<0$.

Case 2. Assume that $u_{1}$ and $u_{2}$ have no common point, that is $v(t) \neq 0$ on $[0, T]$. We may assume that $v>0$ on $[0, T]$.

(i) Let $v^{\prime}=0$ on $[0, T]$. Then, by (4.1a) and (4.2),

$$
v^{\prime \prime}(t)>\frac{a}{t} v^{\prime}(t)-h_{\mathcal{K}}(t)\left|v^{\prime}(t)\right|=0 \text { for a.e. } t \in[0, T]
$$


in contradiction to $v^{\prime \prime}=0$ on $[0, T]$.

(ii) Let $v^{\prime}\left(t_{1}\right) \neq 0$ for some $t_{1} \in(0, T)$. If $v^{\prime}\left(t_{1}\right)>0$, then we can find an interval $[\alpha, \beta] \subset\left(t_{1}, T\right]$ satisfying (4.4). If $v^{\prime}\left(t_{1}\right)<0$, then we can find an interval $[\alpha, \beta] \subset\left[0, t_{1}\right)$ satisfying $(4.5)$.

The above discussion shows that the existence of $[\alpha, \beta]$ satisfying either (4.4) or (4.5) leads to a contradiction. Hence, the solution $u_{1}$ and $u_{2}$ are equal which completes the proof.

Theorem 4.3. (Existence) Let $a<0$ and let us assume (2.2) to hold. Moreover, assume that there exist $A, B \in \mathbb{R}, A \leq B, c>0, \omega \in C[0, \infty)$, and $\psi \in L_{1}[0, T]$ such that the following conditions hold:

$$
f(t, A, 0) \leq 0, \quad f(t, B, 0) \geq 0
$$

for a.e. $t \in[0, T]$,

$$
f(t, x, y) \text { sign } y \leq \omega(|y|)(|y|+\psi(t))
$$

for a.e. $t \in[0, T]$ and all $x \in[A, B], y \in \mathbb{R}$, where

$$
\omega(x) \geq c, \quad x \in[0, \infty), \quad \int_{0}^{\infty} \frac{\mathrm{d} s}{\omega(s)}=\infty .
$$

Then problem (4.1) has a solution $u$ such that

$$
A \leq u(t) \leq B, \quad t \in[0, T]
$$

Proof. Step 1. Existence of auxiliary solutions $u_{n}$.

Let

$$
r:=\|\psi\|_{1}+\left(1+\frac{T}{c}\right)(B-A) .
$$

Then, by (4.8), there exists $\rho^{*}>0$ such that

$$
\int_{0}^{\rho^{*}} \frac{\mathrm{d} s}{\omega(s)}>r
$$

For $y \in \mathbb{R}$, let

$$
\chi(y)= \begin{cases}1 & \text { if }|y| \leq \rho^{*}, \\ 2-\frac{|y|}{\rho^{*}} & \text { if } \rho^{*}<|y|<2 \rho^{*}, \\ 0 & \text { if }|y| \geq 2 \rho^{*} .\end{cases}
$$

Without loss of generality we can assume that $\frac{1}{n}<T$ for each $n \in \mathbb{N}$. Otherwise $\mathbb{N}$ is replaced by $\mathbb{N}^{\prime}=\left\{n \in \mathbb{N}: \frac{1}{n}<T\right\}$. Motivated by [17], we choose $n \in \mathbb{N}$ and, for a.e. $t \in[0, T]$, all $x, y \in \mathbb{R}, \varepsilon \in[0,1]$, we define

$$
h_{n}(t, x, y):= \begin{cases}\chi(y)\left(\frac{a}{t} y+f(t, x, y)\right)-\frac{A}{n} & \text { if } t \in\left(\frac{1}{n}, T\right], \\ -\frac{A}{n} & \text { if } t \in\left[0, \frac{1}{n}\right],\end{cases}
$$




$$
\begin{aligned}
& \omega_{A}(t, \varepsilon):=\sup \left\{\left|h_{n}(t, A, 0)-h_{n}(t, A, y)\right|:|y| \leq \varepsilon\right\}, \\
& \omega_{B}(t, \varepsilon):=\sup \left\{\left|h_{n}(t, B, 0)-h_{n}(t, B, y)\right|:|y| \leq \varepsilon\right\}, \\
& f_{n}(t, x, y):= \begin{cases}h_{n}(t, B, y)+\omega_{B}\left(t, \frac{x-B}{x-B+1}\right) & \text { if } x>B, \\
h_{n}(t, x, y) & \text { if } A \leq x \leq B, \\
h_{n}(t, A, y)-\omega_{A}\left(t, \frac{A-x}{A-x+1}\right) & \text { if } x<A .\end{cases}
\end{aligned}
$$

It can be shown that $w_{A}$ and $w_{B}$ satisfy the $L_{p}$-Carathéodory conditions on $[0, T] \times[0,1]$, are nondecreasing in their second argument and $w_{A}(t, 0)=w_{B}(t, 0)=$ 0 a.e. on $[0, T]$, see [17]. Therefore, $f_{n}$ also satisfies the $L_{p}$-Carathéodory conditions on $[0, T] \times \mathbb{R} \times \mathbb{R}$ and there exists a function $m_{n} \in L_{p}[0, T]$ such that

$$
\left|f_{n}(t, x, y)\right| \leq m_{n}(t) \text { for a.e. } t \in[0, T] \text { and all }(x, y) \in \mathbb{R} \times \mathbb{R} \text {. }
$$

Note that $h_{n}$ can be written in the form

$$
h_{n}(t, x, y)=\mu_{n}(t) \chi(y)\left(\frac{a}{t} y+f(t, x, y)\right)-\frac{A}{n},
$$

where

$$
\mu_{n}(t)=\left\{\begin{array}{l}
0 \text { if } t \in\left[0, \frac{1}{n}\right] \\
1 \text { if } t \in\left(\frac{1}{n}, T\right]
\end{array}\right.
$$

We can see that

$$
\frac{A}{n}+h_{n}(t, A, 0) \leq 0, \frac{B}{n}+h_{n}(t, B, 0) \geq 0 \quad \text { for a.e. } t \in[0, T] .
$$

Consider the auxiliary regular Neumann problem (4.12), (3.1), where

$$
u^{\prime \prime}=\frac{u}{n}+f_{n}\left(t, u, u^{\prime}\right) .
$$

It is easy to verify that the homogeneous problem

$$
u^{\prime \prime}=\frac{u}{n}, \quad u^{\prime}(0)=0, \quad u^{\prime}(T)=0
$$

has only the trivial solution. Hence, by (4.10) and the Fredholm-like existence theorem (see e.g. [22] or [27]), there exists a solution $u_{n} \in A C^{1}[0, T]$ of problem (4.12), (4.1b) for all $n \in \mathbb{N}$.

Step 2. Estimates of $u_{n}$.

Now, we prove that

$$
A \leq u_{n}(t) \leq B, t \in[0, T], n \in \mathbb{N}
$$

Let us define $v:=A-u_{n}$ and assume

$$
\max \{v(t): t \in[0, T]\}=v\left(t_{0}\right)>0 \text {. }
$$


Then $v^{\prime}\left(t_{0}\right)=0$, which is clear if $t_{0} \in(0, T)$, or it follows from (4.1b) if $t_{0} \in\{0, T\}$. First, assume that $t_{0} \in[0, T)$. Then we can find $\delta>0$ such that

$$
v(t)>0, \quad\left|v^{\prime}(t)\right|=\left|u_{n}^{\prime}(t)\right|<\frac{v(t)}{v(t)+1}<1 \text { for } t \in\left[t_{0}, t_{0}+\delta\right] \subset[0, T]
$$

Consequently,

$$
\begin{aligned}
u_{n}^{\prime \prime}(t) & =f_{n}\left(t, u_{n}(t), u_{n}^{\prime}(t)\right)+\frac{u_{n}(t)}{n}=h_{n}\left(t, A, u_{n}^{\prime}(t)\right)-\omega_{A}\left(t, \frac{v(t)}{v(t)+1}\right)+\frac{u_{n}(t)}{n} \\
& \leq h_{n}(t, A, 0)+h_{n}\left(t, A, u_{n}^{\prime}(t)\right)-h_{n}(t, A, 0)-\omega_{A}\left(t,\left|u_{n}^{\prime}(t)\right|\right)+\frac{u_{n}(t)}{n} \\
& \leq h_{n}(t, A, 0)+\frac{A-v(t)}{n} \\
& <0 \text { for } t \in\left[t_{0}, t_{0}+\delta\right] .
\end{aligned}
$$

Hence

$$
0>\int_{t_{0}}^{t} u_{n}^{\prime \prime}(s) \mathrm{d} s=u_{n}^{\prime}(t)-u_{n}^{\prime}\left(t_{0}\right)=u_{n}^{\prime}(t)=-v^{\prime}(t) \text { for } t \in\left(t_{0}, t_{0}+\delta\right],
$$

which contradicts (4.14). Assume that $t_{0}=T$. Then we can find $\delta>0$ such that

$$
v(t)>0, \quad\left|v^{\prime}(t)\right|=\left|u_{n}^{\prime}(t)\right|<\frac{v(t)}{v(t)+1}<1 \quad \text { for } t \in[T-\delta, T] \subset[0, T] .
$$

Then, as above, we have

$$
u_{n}^{\prime \prime}(t) \leq h_{n}(t, A, 0)+\frac{A-v(t)}{n}<0 \text { for } t \in[T-\delta, T] .
$$

Hence

$$
0>\int_{t}^{T} u_{n}^{\prime \prime}(s) \mathrm{d} s=-u_{n}^{\prime}(t)=v^{\prime}(t) \text { for } t \in[T-\delta, T),
$$

in a contrary to (4.14).

Now, let $z:=u_{n}-B$ and assume

$$
\max \{z(t): t \in[0, T]\}=z(\xi)>0 .
$$

Then $z^{\prime}(\xi)=0$, where $\xi \in[0, T]$. Assume that $\xi \in[0, T)$. Then we can find $\delta>0$ such that

$$
z(t)>0, \quad\left|z^{\prime}(t)\right|=\left|u_{n}^{\prime}(t)\right|<\frac{z(t)}{z(t)+1}<1 \text { for } t \in[\xi, \xi+\delta] \subset[0, T] .
$$


Consequently,

$$
\begin{aligned}
u_{n}^{\prime \prime}(t) & =f_{n}\left(t, u_{n}(t), u_{n}^{\prime}(t)\right)+\frac{u_{n}(t)}{n}=h_{n}\left(t, B, u_{n}^{\prime}(t)\right)+\omega_{B}\left(t, \frac{z(t)}{z(t)+1}\right)+\frac{u_{n}(t)}{n} \\
& \geq h_{n}(t, B, 0)+h_{n}\left(t, B, u_{n}^{\prime}(t)\right)-h_{n}(t, B, 0)+\omega_{B}\left(t,\left|u_{n}^{\prime}(t)\right|\right)+\frac{u_{n}(t)}{n} \\
& \geq h_{n}(t, B, 0)+\frac{B+z(t)}{n} \\
& >0 \text { for } t \in[\xi, \xi+\delta] .
\end{aligned}
$$

Then

$$
0<\int_{t_{0}}^{t} u_{n}^{\prime \prime}(s) \mathrm{d} s=u_{n}^{\prime}(t)=z^{\prime}(t) \text { for } t \in(\xi, \xi+\delta],
$$

which contradicts (4.15). If $\xi=T$, then there exists $\delta>0$ such that

$$
z(t)>0, \quad\left|z^{\prime}(t)\right|=\left|u_{n}^{\prime}(t)\right|<\frac{z(t)}{z(t)+1}<1 \text { for } t \in[T-\delta, T] \subset[0, T]
$$

Arguing as above we have

$$
u_{n}^{\prime \prime}(t)>0 \text { for } t \in[T-\delta, T] .
$$

Then

$$
0<\int_{t}^{T} u_{n}^{\prime \prime}(s) \mathrm{d} s=-u_{n}^{\prime}(t)=-z^{\prime}(t) \text { for } t \in[T-\delta, T),
$$

contradicting (4.15). Consequently, we have shown that (4.14) holds.

Step 3. Estimates of $u_{n}^{\prime}$.

We now show that

$$
\left|u_{n}^{\prime}(t)\right| \leq \rho^{*}, t \in[0, T], n \in \mathbb{N} .
$$

Due to (4.11) and (4.12),

$$
\begin{aligned}
& u_{n}^{\prime \prime}(t) \operatorname{sign} u_{n}^{\prime}(t) \\
& \quad=\left\{\mu_{n}(t) \chi\left(u_{n}^{\prime}(t)\right)\left(\frac{a}{t} u_{n}^{\prime}(t)+f\left(t, u_{n}(t), u_{n}^{\prime}(t)\right)\right)+\frac{u_{n}(t)-A}{n}\right\} \operatorname{sign} u_{n}^{\prime}(t)
\end{aligned}
$$

for a.e. $t \in[0, T]$ and all $n \in \mathbb{N}$. Denote $\rho:=\left\|u_{n}^{\prime}\right\|_{\infty}=\left|u_{n}^{\prime}\left(t_{0}\right)\right|$ and assume $\rho>0$. Then $t_{0} \in(0, T)$. In the following part of the proof, we discuss two cases, $u_{n}^{\prime}\left(t_{0}\right)=\rho$ and $u_{n}^{\prime}\left(t_{0}\right)=-\rho$.

Case 1. Let $u_{n}^{\prime}\left(t_{0}\right)=\rho$. Then there exists $t_{1} \in\left[0, t_{0}\right)$ such that $u_{n}^{\prime}(t)>0$ on 
$\left(t_{1}, t_{0}\right]$ and $u_{n}^{\prime}\left(t_{1}\right)=0$. By $(4.7)$ and (4.17), we obtain

$$
\begin{aligned}
u_{n}^{\prime \prime}(t) & =\mu_{n}(t) \chi\left(u_{n}^{\prime}(t)\right)\left(\frac{a}{t} u_{n}^{\prime}(t)+f\left(t, u_{n}(t), u_{n}^{\prime}(t)\right)\right)+\frac{u_{n}(t)-A}{n} \\
& \leq \mu_{n}(t) \chi\left(u_{n}^{\prime}(t)\right) f\left(t, u_{n}(t), u_{n}^{\prime}(t)\right)+\frac{u_{n}(t)-A}{n} \\
& \leq \omega\left(u_{n}^{\prime}(t)\right)\left(u_{n}^{\prime}(t)+\psi(t)\right)+\frac{B-A}{n} \\
& \leq \omega\left(u_{n}^{\prime}(t)\left(u_{n}^{\prime}(t)+\psi(t)+\frac{B-A}{c}\right)\right.
\end{aligned}
$$

for a.e. $t \in\left[t_{1}, t_{0}\right]$. In particular,

$$
\frac{u_{n}^{\prime \prime}(t)}{\omega\left(u_{n}^{\prime}(t)\right)} \leq u_{n}^{\prime}(t)+\psi(t)+\frac{B-A}{c} \text { for a.e. } t \in\left[t_{1}, t_{0}\right]
$$

Hence

$$
\int_{t_{1}}^{t_{0}} \frac{u_{n}^{\prime \prime}(t)}{\omega\left(u_{n}^{\prime}(t)\right)} \mathrm{d} t \leq \int_{t_{0}}^{t_{1}}\left(u_{n}^{\prime}(t)+\psi(t)+\frac{B-A}{c}\right) \mathrm{d} t
$$

and

$$
\int_{0}^{\rho} \frac{\mathrm{d} t}{\omega(t)}<u_{n}\left(t_{1}\right)-u_{n}\left(t_{0}\right)+\|\psi\|_{1}+\frac{(B-A) T}{c} \leq\left(1+\frac{T}{c}\right)(B-A)+\|\psi\|_{1}=r .
$$

Therefore

$$
\int_{0}^{\rho} \frac{\mathrm{d} t}{\omega(s)}<r
$$

and $\rho<\rho^{*}$ follows.

Case 2. Let $u_{n}^{\prime}\left(t_{0}\right)=-\rho$. Then there exists $t_{1} \in\left[0, t_{0}\right)$ such that $u_{n}^{\prime}(t)<0$ on $\left(t_{1}, t_{0}\right]$ and $u_{n}^{\prime}\left(t_{1}\right)=0$. By (4.7) and (4.17), we deduce

$$
\begin{aligned}
-u_{n}^{\prime \prime}(t) & =-\mu_{n}(t) \chi\left(u_{n}^{\prime}(t)\right)\left(\frac{a}{t} u_{n}^{\prime}(t)+f\left(t, u_{n}(t), u_{n}^{\prime}(t)\right)\right)-\frac{u_{n}(t)-A}{n} \\
& \leq-\mu_{n}(t) \chi\left(u_{n}^{\prime}(t)\right) f\left(t, u_{n}(t), u_{n}^{\prime}(t)\right) \\
& \leq \omega\left(-u_{n}^{\prime}(t)\right)\left(-u_{n}^{\prime}(t)+\psi(t)\right)
\end{aligned}
$$

for a.e. $t \in\left[t_{1}, t_{0}\right]$. In particular,

$$
-\frac{u_{n}^{\prime \prime}(t)}{\omega\left(-u_{n}^{\prime}(t)\right)} \leq-u_{n}^{\prime}(t)+\psi(t) \text { for a.e. } t \in\left[t_{1}, t_{0}\right]
$$

and

$$
-\int_{t_{1}}^{t_{0}} \frac{u_{n}^{\prime \prime}(t)}{\omega\left(-u_{n}^{\prime}(t)\right)} \mathrm{d} t \leq \int_{t_{0}}^{t_{1}}\left(\psi(t)-u_{n}^{\prime}(t)\right) \mathrm{d} t
$$

Hence

$$
\int_{0}^{\rho} \frac{\mathrm{d} t}{\omega(s)} \leq\|\psi\|_{1}+u_{n}\left(t_{0}\right)-u_{n}\left(t_{1}\right) \leq\|\psi\|_{1}+B-A<r
$$


Consequently,

$$
\int_{0}^{\rho} \frac{\mathrm{d} t}{\omega(s)}<r
$$

which implies $\rho<\rho^{*}$. Hence (4.16) holds.

Step 4. Convergence of $\left\{u_{n}\right\}$.

By (4.13) and (4.16), $\left\{u_{n}\right\}$ is bounded in $C^{1}[0, T]$. Since $f$ is $L_{p}$-Carathéodory on $[0, T] \times \mathbb{R}^{2}$, there exists $m \in L_{p}[0, T]$ such that

$$
\left|f\left(t, u_{n}(t), u_{n}^{\prime}(t)\right)\right| \leq m(t) \text { for a.e. } t \in[0, T] \text { and all } n \in \mathbb{N} .
$$

Choose $b \in(0, T]$. Then there exists $n_{0} \in \mathbb{N}$ such that $[b, T] \subset\left[\frac{1}{n}, T\right]$ for all $n \geq n_{0}$. Hence

$$
\begin{aligned}
u_{n}^{\prime}(t) & =-\int_{t}^{T}\left(f_{n}\left(t, s, u_{n}(s), u_{n}^{\prime}(s)\right)+\frac{u_{n}(s)}{n}\right) \mathrm{d} s \\
& =-\int_{t}^{T}\left(f\left(s, u_{n}(s), u_{n}^{\prime}(s)\right)+\frac{a}{s} u_{n}^{\prime}(s)+\frac{u_{n}(s)-A}{n}\right) \mathrm{d} s
\end{aligned}
$$

for $t \in[b, T]$ and $n \geq n_{0}$. Let $b \leq t_{1}<t_{2} \leq T$. Then, by (4.18) and (4.19),

$$
\begin{aligned}
\left|u_{n}^{\prime}\left(t_{2}\right)-u_{n}^{\prime}\left(t_{1}\right)\right| & =\left|\int_{t_{1}}^{t_{2}}\left(f\left(t, u_{n}(t), u_{n}^{\prime}(t)\right)+\frac{a}{t} u_{n}^{\prime}(t)+\frac{u_{n}(t)-A}{n}\right) \mathrm{d} t\right| \\
& \leq \int_{t_{1}}^{t_{2}} m(t) \mathrm{d} t+\left(\frac{|a| \rho^{*}}{b}+B-A\right)\left(t_{2}-t_{1}\right)
\end{aligned}
$$

for $n \geq n_{0}$. Hence $\left\{u_{n}^{\prime}\right\}_{n \geq n_{0}}$ is equicontinuous on $[b, T]$ and since $\left\{u_{n}\right\}$ is bounded in $C^{1}[0, T]$, the Arzelà-Ascoli theorem and the diagonalization theorem (see e.g. [27]) guarantee that there exist a subsequence $\left\{u_{\ell}\right\}$ of $\left\{u_{n}\right\}$ and $u \in C[0, T] \cap$ $C^{1}(0, T]$ such that

$$
\begin{aligned}
& \lim _{\ell \rightarrow \infty} u_{\ell}(t)=u(t) \quad \text { uniformly on }[0, T], \\
& \lim _{\ell \rightarrow \infty} u_{\ell}^{\prime}(t)=u^{\prime}(t) \quad \text { locally uniformly on }(0, T] .
\end{aligned}
$$

Clearly $u^{\prime}(T)=0$. By (4.13) and (4.16)

$$
A \leq u(t) \leq B \text { for } t \in[0, T], \quad\left|u^{\prime}(t)\right| \leq \rho^{*} \text { for } t \in(0, T] .
$$

Hence (3.3) holds. Passing to the limit as $\ell \rightarrow \infty$ in (4.19), where $u_{n}$ is replaced by $u_{\ell}$, we obtain

$$
u^{\prime}(t)=-\int_{t}^{T}\left(f\left(s, u(s), u^{\prime}(s)\right)+\frac{a}{s} u^{\prime}(s)\right) \mathrm{d} s \text { for } t \in(0, T]
$$

by the Lebesgue dominated convergence theorem. Hence the limit function $u$ belongs to $A C_{l o c}^{1}(0, T]$ and solves equation (4.1a) a.e. on $[0, T]$. The local uniform 
convergence of $\left\{u_{\ell}^{\prime}\right\}$ on $(0, T]$ does not guarantee $u^{\prime}(0)=0$. However, we can apply Corollary 3.6 to find out that $u \in A C^{1}[0, T]$ and $u^{\prime}(0)=0$. Therefore, $u$ satisfies the Neumann conditions (4.1b). We see that $u$ is a solution of problem (4.1) such that $A \leq u \leq B$ on $[0, T]$ which completes the proof.

Example 4.4. Let $T=1$. For $t \in(0,1], x, y \in \mathbb{R}$, choose

$$
f(t, x, y)=\frac{1}{\sqrt{1-t}}\left(3 x\left(x^{2}-1\right)+e^{x} y\right)-\cos (3 \pi t) .
$$

Then Theorem 4.3 can be applied to $f$ and for both $A=-5 / 4, B=-1 / 2$ and $A=1 / 2, B=5 / 4$. Consequently, problem (4.1) with $f$ given by (4.21), has two solutions $u_{1}$ and $u_{2}$ satisfying

$$
-\frac{5}{4} \leq u_{1}(t) \leq-\frac{1}{2}, \quad \frac{1}{2} \leq u_{2}(t) \leq \frac{5}{4}, t \in[0,1]
$$

The existence of two different solutions $u_{1}$ and $u_{2}$ corresponds to the fact that $f$ does not satisfy condition (4.2) of Theorem 4.2 .

The next theorem for the existence of a unique solution of the Neumann problem (4.1) follows immediately from Theorems 4.2 and 4.3.

Theorem 4.5. (Existence and uniqueness) Let all assumptions of Theorem 4.2 and Theorem 4.3 be satisfied. Then problem (4.1) has a unique solution $u$. This solution satisfies (4.9).

Example 4.6. The following function,

$$
f(t, x, y)=\frac{1}{\sqrt{1-t}}\left(x^{3}-y^{5}\right)-10 \sin (4 \pi t)
$$

$t \in(0,1], x, y \in \mathbb{R}$, satisfies the assumptions of Theorem 4.5 for $A=-10^{1 / 3}$ and $B=10^{1 / 3}$. Therefore problem (4.1) with $f$ given by (4.22) has a unique solution.

\section{$5 \quad$ Numerical Simulation}

To illustrate the solution behavior, described by Theorems 4.3 and 4.5 we carried out a series of numerical calculations using a MATLAB ${ }^{\mathrm{TM}}$ software package bvpsuite designed to solve boundary value problems in ordinary differential equations and differential algebraic equations. The solver is based on a class of collocation method (including methods of different orders). The code also provides the asymptotically correct estimate for the error of the numerical approximation. To enhance the efficiency the code attempts to solve the problem on a mesh adapted to the solution behavior, in such a way that the tolerance is 
satisfied with the least possible effort. Error estimate procedure and the mesh adaptation work dependably provided that the solution of the problem is appropriately smooth ${ }^{1}$. The software and the manual with a short description of the code can be downloaded from http://www.math.tuwien.ac.at/ ewa. Further information can be found in [18] and [20]. This software has already been used for a variety of singular boundary value problems relevant for applications, see e.g. [25].

We discuss Neumann problems of the form,

$$
\begin{aligned}
& u^{\prime \prime}(t)=\frac{a}{t} u^{\prime}(t)+\frac{1}{\sqrt{1-t}}\left(3 u(t)\left(u^{2}(t)-1\right)+e^{u(t)} u^{\prime}(t)\right)-\cos (3 \pi t), \\
& u^{\prime}(0)=u^{\prime}(1)=0
\end{aligned}
$$

and

$$
\begin{aligned}
& u^{\prime \prime}(t)=\frac{a}{t} u^{\prime}(t)+\frac{1}{\sqrt{1-t}}\left(u^{3}(t)-u^{\prime 5}(t)\right)-10 \sin (4 \pi t), \\
& u^{\prime}(0)=u^{\prime}(1)=0
\end{aligned}
$$

cf. Examples 4.4 and 4.6, respectively. All solutions were computed on the unit interval $[0,1]$.
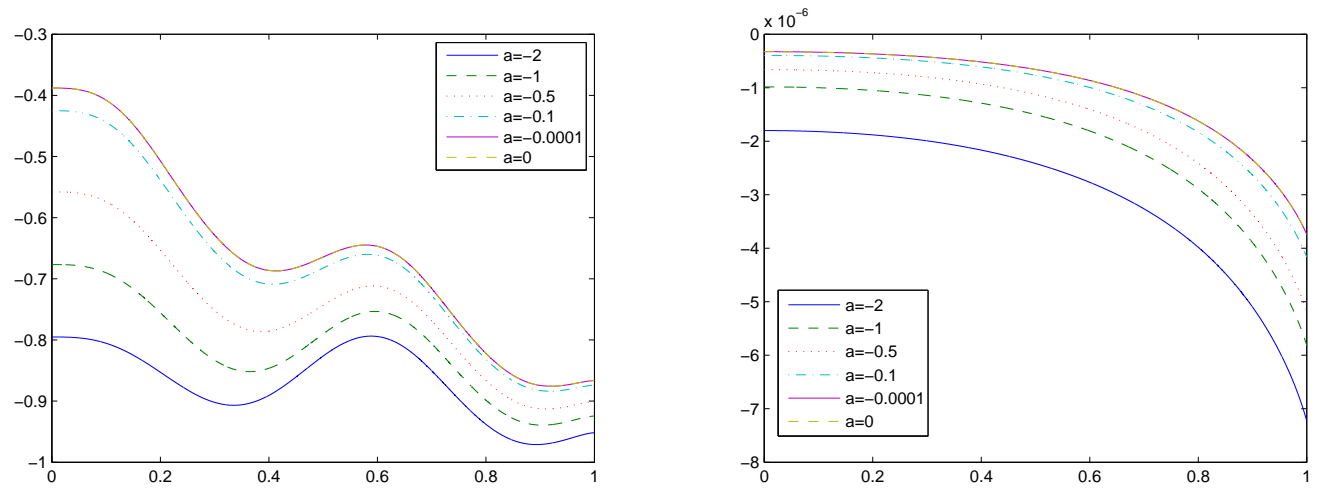

Figure 1: Illustrating Theorem 4.3: Solutions $-5 / 4 \leq u_{1}(t) \leq-1 / 2$ of problem (5.1) for different values of $a$ (left), and the related error estimates (right). The initial solution for the Newton iteration was $u_{0}(t) \equiv-1$; the number of mesh points used $N=1000$.

\footnotetext{
${ }^{1}$ The required smoothness of higher derivatives is related to the order of the used collocation method.
} 

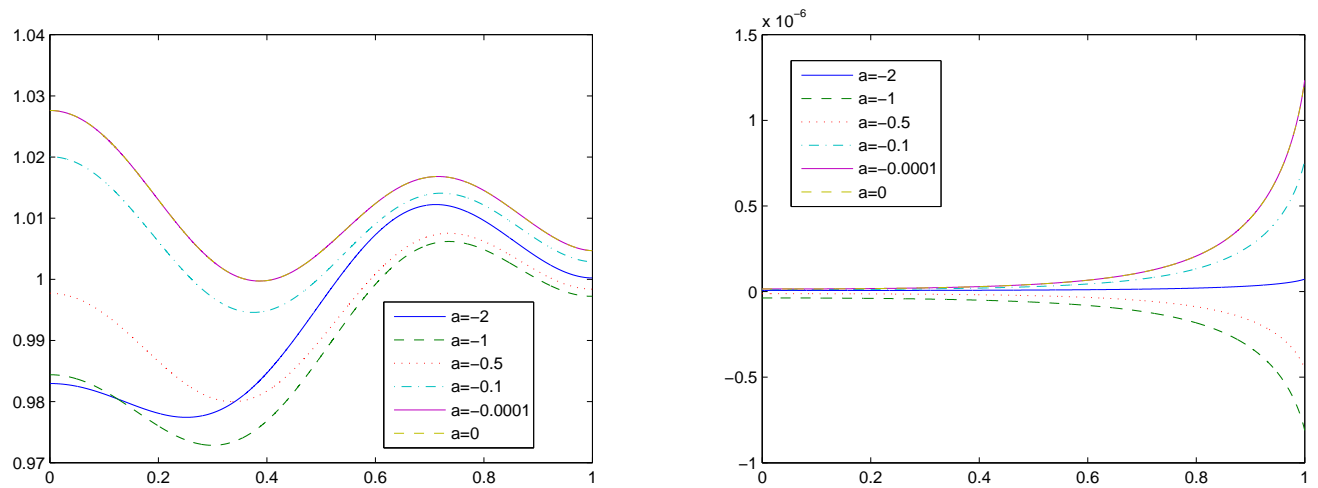

Figure 2: Illustrating Theorem 4.3: Solutions $1 / 2 \leq u_{1}(t) \leq 5 / 4$ of problem (5.1) for different values of $a$ (left), and the related error estimates (right). The initial solution for the Newton iteration was $u_{0}(t) \equiv 1$; the number of mesh points used $N=1000$.

As shown in Figures 1 and 2, we could find two different solutions $u_{1}$ and $u_{2}$ lying in regions indicated in Example 4.4. Recall that Theorem 4.3 guaranties the existence of a solution to a Neumann problem but not its uniqueness. Since in this case the solution is very unsmooth the mesh adaptation strategy does not work properly and therefore the calculations have been carried out on an equidistant mesh containing 1000 mesh points. We doubled the number of mesh points to provide a rough error estimate for the global error of the approximation. According to Theorem 4.5, the solution of problem (5.2a) is unique, cf. Figure 3.
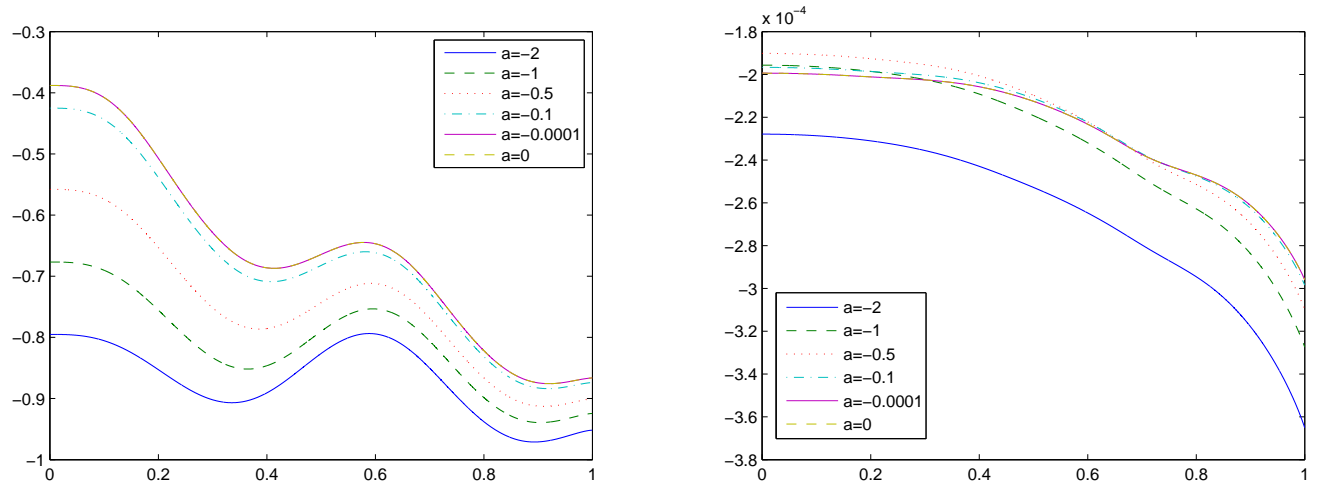

Figure 3: Illustrating Theorem 4.5: Solutions of problem (5.2a) for different values of $a$ (left), and the related error estimates (right). The initial solution for the Newton iteration was $u_{0}(t) \equiv 1$; the number of mesh points used $N=1000$. 


\section{Acknowledgements}

This research was supported by the Council of Czech Goverment MSM6198959214, and by the grant No. A100190703 of the Grant Agency of the Academy of Sciences of the Czech Republic.

\section{References}

[1] R.P. Agarwal, And D. O'Regan. Singular problems arising in circular membrane theory. Dyn. Contin. Discrete Impuls. Syst., Ser. A Math. Anal. 10, No. 6, (2003), 965-972.

[2] J.V. BAXLEY. A singular nonlinear boundary value problem: membrane response of a spherical cap. SIAM J. Appl. Math. 48 (1988), 497-505.

[3] J.V. Baxley, And G.S. GersdorfF. Singular reaction-diffusion boundary value problem. J. Differential Equations 115 (1995), 441-457.

[4] C. Budd, O. Koch, And E. Weinmüller. Computation of Self-similar Solution Profiles for the Nonlinear Schrödinger Equation. Computing 77 (2006), 335-346.

[5] C. Budd, O. Koch, And E. Weinmüller. From nonlinear PDEs to singular ODEs. Appl. Num. Math. 56 (2006), 413-422.

[6] A. Constantin. Sur un probleme aux limites en mecanique non lineaire. C. R. Acad. Sci. Paris 320, Serie I (1995), 1465-1468.

[7] R.W. Dickey. Rotationally symmetric solutions for shallow membrane caps. Quart. Appl. Math. 47 (1989), 571-581.

[8] R.W. Dickey. The plane circular elastic surface under normal pressure. Archs. Ration. Mech. Analysis 26 (1967), 219-236.

[9] R. Hammerling, O. Koch, C. Simon, and E. Weinmüller. Numerical Solution of Eigenvalue Problems in Electronic Structure Computations. In preparation.

[10] R. Hammerling, O. Koch, C. Simon, and E. Weinmüller. Numerical Solution of Singular Eigenvalue Problems in ODEs with a Focus on Problems Posed on Semi-Infinite Intervals. In preparation.

[11] V. HlavaceK, M. Marek, And M. KubiceK. Modeling of chemical reactors-X. Multiple solutions of enthalpy and mass balances for a catalytic reaction within a porous catalyst particle. Chemical Eng. Science 23 (1968), 1083-1097. 
[12] F. DE Hoog, AND R. WeIss. Difference methods for boundary value problems with a singularity of the first kind. SIAM J. Numer. Anal. 13 (1976), 775-813.

[13] F. DE Hoog, And R. Weiss. The numerical solution of boundary value problems with an essential singularity. SIAM J. Numer. Anal. 16 (1979), 637-669.

[14] F. DE Hoog, And R. Weiss. The application of Runge-Kutta schems to singular initial value problems. Math. Comp. 44 (1985), 93-103.

[15] F. De Hoog, And R. WeIss. Collocation methods for singular boundary value problems. SIAM J. Numer. Anal. 15 (1978), 198-217.

[16] K.N. Johnson. Circularly symmetric deformation of shallow elastic membrane caps. Quart. Appl. Math. 55 (1997), 537-550.

[17] I.T. Kiguradze, AND B.L. Shekhter. Singular boundary value problems for second order ordinary differential equations. Itogi Nauki i Techniki, Ser. Sovrem. Probl. Mat. Nov. Dost. 30 (1987), 105-201 (in Russian), translated in J. Sovier. Math. 43 (1988), 2340-2417.

[18] G. Kitzhofer Numerical Treatment of Implicit Singular BVPs, Ph.D. Thesis Inst. for Anal. and Sci. Comput., Vienna University of Technology, Austria. In preparation.

[19] G. Kitzhofer, O. Koch, P. Lima, And E. Weinmüller. Efficient Numerical Solution of the Density Profile Equation in Hydrodynamics. J. Sci. Comp. DOI: 10.1007/10915-005-9020-5 (2006).

[20] G. Kitzhofer, G. Pulverer, C. Simon, O. Koch, And E. Weinmüller. The New Matlab Solver BVPSUITE for the Solution of Singular Implicit BVPs. In preparation.

[21] O. KосH. Asymptotically correct error estimation for collocation methods applied to singular boundary value problems. Numer. Math. 101 (2005), 143164.

[22] A. LAsota. Sur les problèmes linéaires aux limites pour un système d'équations différentielles ordinaires. Bull. Acad. Polon. Sci. Sér. Sci. Math. Astron. Phys. 10 (1962), 565-570.

[23] J.W. LEe, AND D. O'REGAN. Existence of solutions to some initial value, two-point, and multi-point voundary value problems with discontinuous nonlinearities. Appl. Anal. 33 (1989), 57-77. 
[24] I. Rachůnková, O. Koch, G. Pulverer, and E. Weinmüller. On a singular bounadry value problem arising in the theory of shallow membrane caps. J. Math. Anal. Appl. 332 (2007), 523-541.

[25] I. Rachunkova, G. Pulverer, E. B. Weinmller. A unified approach to singular problems arising in the membran theory. To appear in Math. Anal. and Appl.

[26] I. Rachůnková, S. StaněK, And M. Tvrdý. Singularities and Laplacians in Boundary Value Problems for Nonlinear Ordinary Differential Equations. Handbook of Differential Equations. Ordinary Differential Equations, Ed. by A. Caňada, P. Drábek, A. Fonda, Vol. 3. Elsevier (2006), 607-723.

[27] I. Rachůnková, S. Staněk, And M. Tvrdý. Solvability of Nonlinear Singular Problems for Ordinary Differential Equations. Hindawi Publ. Corp., New York 2008.

[28] I. Rachưnková, S. StaněK, E. Weinmüller, And M. Zenz. Limit properties of solutions of singular second-order differential equations. Boundary Value Problems, Volume 2009 (2009), Article ID 905769, doi:10.1155/2009/905769, 28 pages.

[29] J.Y. SHIN. A singular nonlinear differential equation arising in the Homann flow. J. Math. Anal. Appl. 212 (1997), 443-451.

[30] E. WeInMüLleR. On the boundary value problems for systems of ordinary second order differential equations with a singularity of the first kind. SIAM J. Math. Anal. 15 (1984), 287-307.

[31] E. Weinmüller. Collocation for singular boundary value problems of second order. SIAM J. Numer. Anal. 23 (1986), 1062-1095. 